\title{
Experimental infection with neuropathogenic equid herpesvirus type 1 (EHV-1) in adult horses
}

\author{
Lutz S. Goehring a,*, C. van Maanen ${ }^{\mathrm{b}}$, Marcel Berendsen ${ }^{\mathrm{c}}$, Ann Cullinane ${ }^{\mathrm{d}}$, Raoul J. de Groot ${ }^{\mathrm{e}}$, \\ Peter J.M. Rottier ${ }^{\mathrm{e}}$, Jeroen J.C.M. Wesselingh ${ }^{\mathrm{f}}$, Marianne M. Sloet van Oldruitenborgh-Oosterbaan ${ }^{\mathrm{f}}$ \\ ${ }^{a}$ Department of Clinical Sciences, College of Veterinary Medicine and Biomedical Sciences, Colorado State University, 300 West Drake Road, Fort Collins, Colorado 80523, USA \\ ${ }^{\mathrm{b}}$ Animal Health Service, Department of Diagnostic Research and Epidemiology, PO Box 9, 7400 AA Deventer, The Netherlands \\ ${ }^{\mathrm{c}}$ Burg. van Heemstrakwartier 113, 3731 TC De Bilt, The Netherlands \\ ${ }^{\mathrm{d}}$ Virology Unit, The Irish Equine Centre, Johnstown, Naas, County Kildare, Ireland \\ ${ }^{\mathrm{e}}$ Virology Division, Department of Infectious Diseases and Immunology, Faculty of Veterinary Medicine, and Institute of Biomembranes, Utrecht University, \\ Yalelaan 1, 3584 CL Utrecht, The Netherlands \\ ${ }^{\mathrm{f}}$ Department of Equine Science, Internal Medicine, Faculty of Veterinary Medicine, Utrecht University, Yalelaan 114, 3584 CM Utrecht, The Netherlands
}

\section{A R T I C L E I N F O}

Article history:

Accepted 4 August 2009

\section{Keywords:}

Neuropathogenic EHV-1

Infection experiment

Cerebrospinal fluid analysis

Adult horse

\begin{abstract}
A B S T R A C T
Equid herpesvirus type 1 (EHV-1)-associated myeloencephalopathy (EHM) may follow an infection with the virus in horses. This study tested three hypotheses: (1) a large inhaled dose of a neuropathogenic EHV-1 strain would induce a cell-associated viraemia in all infected horses; (2) neurological disease will only occur in viraemic horses, and (3) the cerebrospinal fluid (CSF) composition following EHV-1 viraemia will be an indicator for EHM.

Four EHV-1 seronegative horses were inoculated with EHV-1 by inhalation. Three developed clinical signs of neurological disease, which were mild in two horses and lacking typical EHM histopathological findings, but moderately severe in the third horse. This latter animal was the only one found to be viraemic, with xanthochromic CSF and spinal cord histopathology findings characteristic of EHM. This study showed that cell-associated viraemia was not guaranteed, despite a large-dose inoculation with EHV-1, yet viraemia was probably a pre-requisite for subsequent development of EHM. The histopathological changes used to confirm EHM may be predicted from CSF analysis.
\end{abstract}

(c) 2009 Elsevier Ltd. All rights reserved.

\section{Introduction}

Abortion, neonatal death, and myeloencephalopathy are potential sequelae of equid herpesvirus type 1 (EHV-1) in the horse. Outbreaks of EHV-1-associated myeloencephalopathy (EHM) occur worldwide, but are rare. EHM primarily affects the spinal cord and disease severity ranges from asymmetrical ataxia, often in combination with weakness in one or more limbs, to complete paralysis. Whilst there may be clinical improvement over time, the signs typically remain recognisable for several weeks to months, if not permanently (van Maanen, 2002).

Following infection with EHV-1, the virus first replicates in the epithelium of the respiratory tract. Shortly afterwards, virus can be detected in regional lymph nodes, from which infected lymphocytes and monocytes are released into the bloodstream, causing a cell-associated viraemia. This viraemia in peripheral blood mononuclear cells (PBMC) is central to the pathogenesis of EHM and leads to a restricted endothelial cell infection of the central nervous

\footnotetext{
* Corresponding author. Tel.: +1 970297 4246; fax: +1 9702971275.

E-mail address: lutz.goehring@colostate.edu (L.S. Goehring).
}

system (CNS) vasculature (the presumed cause of vasculitis and thrombosis) followed by ischaemic degeneration of nervous tissue (Edington et al., 1986; Smith et al., 2001).

Neuropathogenic (np) EHV-1 strains are more often isolated from natural outbreaks of EHM than non-neuropathogenic (nonnp). Neuropathogenic strains differ from non-np strains by a single nucleotide point mutation in the polymerase gene (Nugent et al., 2006). However, when yearling horses were experimentally infected with np strains, they failed to develop neurological disease (Allen and Breathnach, 2006). The duration and magnitude of viraemia were assessed using quantitative PCR analysis, and both aspects of viraemia, duration and magnitude, were found to be significantly higher in the group of np strain-infected horses when compared to the group infected with the non-np strains.

Studies of naturally occurring EHM outbreaks have shown that some breeds and some age groups are at a lower risk of developing EHM than others. Horses younger than 3 years of age do not develop neurological disease, and EHM is more commonly found in Warmblood breeds, Thoroughbred and Standardbred horses, Draught horses and less so in Shetland and Icelandic ponies, Haflingers, and others. In addition, EHM in females and older animals 
tends to be more severe (Goehring et al., 2006a). The percentage of horses with neurological disease during natural outbreaks of EHM in a horse operation is usually 10-20\% (Goehring et al., 2006a). In rare outbreaks, up to $70 \%$ of all horses can develop some degree of neurological disease (van Maanen et al., 2001; Henninger et al., 2007).

A number of factors, both endogenous and exogenous, are involved in EHM occurrence and severity. These factors could include (1) the magnitude and duration of cell-associated viraemia as mediated by strain properties and host immunity, (2) the rate of endothelial cell infection, (3) the host response to vasculopathy, and (4) other unknown factors.

Successful experimental induction of neurological disease has been performed using a combination of infection by the respiratory route with an intramuscular or subcutaneous injection of virus (Jackson et al., 1977; Edington et al., 1986). However, only Mumford et al. (1994) have been able to create a consistent and at times paralytic experimental model of neurological disease by infecting horses that have a low serum antibody titre with a large dose of inhaled EHV-1, strain Ab4, in a nebulised suspension. The severity of neurological disease that they were able to create was dose dependent and strain restricted. Since then, others have tried to induce neurological disease by experimental infection with EHV-1, but with only partial (Goodman et al., 2006) or no success (Goehring et al., 2008).

The character of cerebrospinal fluid (CSF) in horses with acute EHM undergoes substantial changes. The infection damages the blood-brain barrier, allowing erythrocyte leakage into the CSF and development of xanthochromia as the erythrocytes break down. Protein concentrations generally increase because vasculitis at the blood-brain barrier allows albumin to cross from the bloodstream into the CNS and CSF (Donaldson and Sweeney, 1998) thereby causing an increase in the albumin quotient (Andrews et al., 1994).

In a previous study, we successfully placed sub-arachnoidal catheters in two horses to sample CSF twice daily over a period of 17 days (Goehring et al., 2006b). We applied this novel sampling technique during the current experiment for twice daily assessment of CSF, with particular interest in CSF changes during the post-infection period.

The objective of the present pilot study was to create a reliable experimental model of EHV-1-induced neurological disease, with a neuropathogenic strain of the virus isolated from an EHM outbreak with significant neurological case fatality rate (van Maanen et al., 2001), and with virus administered by respiratory tract inoculation. The main hypotheses tested were that (1) a large inhaled dose of an np EHV-1 virus will induce cell-associated viraemia in all infected horses, (2) EHM will only occur in the viraemic horses, and (3) CSF composition following EHV-1 viraemia can aid in predicting whether a horse will develop neurological disease (EHM).

\section{Material and methods}

Pre-experimental period-selection for infection and preparation of horses

Six Standardbred mares, aged 14-20 years, were selected from a pool of 12 Selection was based on the potentially increased risk for developing EHM upon EHV-1 infection in this breed, sex and age group (Goehring et al., 2006a). Further criteria for inclusion in the study were a normal physical and neurological examination and the absence of serological evidence of a recent EHV-1 infection as assessed by an EHV-1 serum neutralisation (SN) test, an EHV-1-specific glycoprotein G (gG) ELISA (Svanovir, Svanova Biotech) and a complement fixation (CF) assay (Hartley et al., 2005). Horses having the lowest measurable SN titres (between $1: 128$ and 1:256) were pre-selected and provisionally included when the absence of antibodies by EHV-1 gG specific ELISA was confirmed.

During the 8 week period between selection and infection, the horses were isolated as a group and underwent daily physical examination. Weekly nasal swabs were collected and assayed for EHV-1 genomes by quantitative PCR analysis (qPCR).
Horses were excluded if nasal discharge, enlarged mandibular lymph nodes or a fe$\operatorname{ver}\left(>38.3^{\circ} \mathrm{C}\right)$ was noticed. One week prior to infection, a CF titre was done and only those animals with a titre of $\leqslant 1: 10$ were allowed to participate.

All six selected horses met the criteria and were allowed to remain in the study. Following the selection period, horses A and B were infected; then, 3 weeks later, horses $C$ and D. The infection was performed at a biosecurity level 3 isolation facility, which was $35 \mathrm{~km}$ away from the original isolation facility. Horses were transported to this location by truck 3 days prior to infection. There, each horse was housed in an individual, completely separated, temperature-controlled stall unit for the complete post-infection observational period of 2 weeks. All four horses were infected under identical conditions and the same sampling protocols were instituted. The two reserve/control horses remained in the original isolation facility until completion of the study. These horses were nebulised with sterile virus culture medium once, followed by daily physical examinations.

During the week prior to the infections, a neurological examination of each horse (A-D) was completed and recorded on video. In addition, sub-arachnoidal, lumbo-sacral catheters (Epidural Pain Management Kit, Mila International) for serial CSF collection were placed (Goehring et al., 2006b); however, this was only achieved in horses A, B, and C. It appeared impossible to place the catheter in horse $\mathrm{D}$ due to a combination of the horse's temperament, anatomical conformation and the unavailability of stocks for additional restraint in the original isolation unit. This horse also appeared extremely nervous, and major changes in its environment prompted prolonged 'weaving', sweating and muscle fasciculations which typically lasted for about $2-3 \mathrm{~h}$

After infection and a 2 week period of observation and sample collection, horses A-D were euthanased and immediately underwent post mortem investigation. The two reserve/control horses were released back into the pool of teaching horses.

\section{Experimental infection on day $0(\mathrm{D} 0)$}

An np EHV-1 strain isolated from a documented, high incidence EHM outbreak in the Netherlands was used for infection (van Maanen et al., 2001). A total inoculation volume of $5 \mathrm{~mL}$ of $10^{7.6} \mathrm{TCID}_{50} / \mathrm{mL}$ virus in virus culture medium (DMEM) was used for each horse. Virus inoculum was prepared as a seventh passage on equine dermal cells. Six millilitres of viral suspension was placed in the holding chamber of a jet nebuliser attached to a face mask (both Trudell Medical International), which allowed closed inhalation of nebulised viral suspension. The infectious dose of the inoculum was determined in $1 \mathrm{~mL}$ of virus suspension recovered from the nebuliser's holding chamber after inoculation was completed.

\section{Clinical evaluation in the post-infection period}

Morning and evening clinical examinations were conducted by two independent veterinarians. The examinations focused on mentation, lung auscultation, the presence of a cough and/or a nasal discharge, palpable aspect of mandibular lymph nodes, presence of fever defined as $\geqslant 38.5^{\circ} \mathrm{C}$, appetite, faecal production and neurological abnormalities. The clinical-neurological examination focused on gait abnormalities described as ataxia, dysmetria and weakness, and on the ability to urinate, tail tone qualities and cranial nerve function which can be a rare sign of EHM (van Maanen, 2002). An adapted version of a scoring system was used to describe neurological abnormalities (Mayhew, 2008).

Neurological dysfunction was determined by scoring dysmetria and ataxia, on a scale of $0-4$ with $0=$ absent; 1 = difficult to detect, visible only with provocation tests; 2 = recognisable on a walk; 3 =obvious truncal sway or marked ataxia; $4=$ very uncoordinated, poor correction. Weakness was also graded on a scale of $0-4$ with $0=$ normal; $0.5=$ decreased tail tone; $1=$ some toe dragging; $2=$ consistent toe dragging; 3 = recumbent horse, but able to rise when assisted; $4=$ unable to stand. Other abnormalities such as single or multiple cranial nerve deficits or dysuria each scored two points. Individual scores were totalled to obtain an overall grade between 0 (no abnormalities) and 10 (highest grade possible). Because a recumbent horse cannot be evaluated for gait characteristics, the maximum grade for weakness (4) would be multiplied by two.

\section{Sample collection and storage}

During the immediate pre- and post-infection period, the following samples were collected: (1) swabs from the nasal passages on the day before inoculation, then every other day; (2) peripheral venous blood up to twice daily (for various purposes as explained later) starting 5 days prior to infection (D-5) and D14 and (3) CSF, twice daily in horses with a sub-arachnoidal catheter system, between D-5 and D14. CSF was collected from all horses from the atlanto-occipital cisterna immediately after euthanasia.

Nasal swabs were placed in empty containers, and snap-frozen in liquid nitrogen followed by initial storage at $-80^{\circ} \mathrm{C}$. After completion of the study, nasal swabs were transferred to a contracted laboratory, where they were mistakenly stored at $-20^{\circ} \mathrm{C}$. Venous blood was collected in plain and EDTA-coated tubes (Vacutainer, BD Diagnostics). About $60 \mathrm{~mL}$ of blood stored in EDTA tubes was used for PBMC isolation, once daily. PBMC were isolated within $4 \mathrm{~h}$ after collection, which was done by routine ficoll-hypaque gradient separation (Lymphoprep, Axis Shields PoC AS) 
(Akens et al., 1997). PBMC were re-suspended in RPMI-1640 medium supplemented with $50 \%$ foetal calf serum and $10 \%$ dimethyl sulfoxide at a concentration of $5 \times 10^{6} / 1.5 \mathrm{~mL}$, then frozen and stored at $-80^{\circ} \mathrm{C}$.

Five millilitres of EDTA blood was used for a complete blood count and differentiation. This sample was spun down immediately after the complete blood count was completed. The plasma fraction was transferred into $1.5 \mathrm{~mL}$ vials and frozen at $-80^{\circ} \mathrm{C}$. Eight millilitres of blood was allowed to clot in plain tubes, then centrifuged $(1000 \mathrm{~g}, 5 \mathrm{~min})$. The serum fraction was transferred into $1.5 \mathrm{~mL}$ vials and frozen at $-80^{\circ} \mathrm{C}$. From horses with a sub-arachnoidal catheter, serum for albumin measurements was collected and stored twice daily. A further vial of serum was stored from all four horses every other day for serology. CSF samples were split into two samples and were stored in EDTA tubes. One sample was immediately spun down at $1000 \mathrm{~g}$ for $5 \mathrm{~min}$; the supernatant was transferred into $1.5 \mathrm{~mL}$ vials and snap-frozen in liquid nitrogen and transferred during the following day into $-80^{\circ} \mathrm{C}$. The second sample was submitted within $4 \mathrm{~h}$ of collection for a cell count and cytology.

Fourteen days post-infection, horses were euthanased with sodium pentobarbital. A full pathological examination immediately followed and all organs were sampled. After the brain and spinal cord were removed from the carcass, $5 \mathrm{~mm}$ slices of spinal cord were taken from the caudal aspect of the cervical, thoracic and lumbar sections and frozen at $-20^{\circ} \mathrm{C}$. The remaining spinal cord tissue was fixed in $4 \%$ formaldehyde. The right trigeminal ganglion, a cut-section of brain stem and half of the rete mirabilis were sampled and stored at $-20{ }^{\circ} \mathrm{C}$, the remainder of the brain was fixed in $4 \%$ formaldehyde. After 7 days of fixation, spinal cord was cut into $10 \mathrm{~cm}$ segments. A $5 \mathrm{~mm}$ slice was cut from the caudal aspect of these sections and prepared for routine histopathology using haematoxylin and eosin stain. When abnormalities were noted in the corresponding histopathological slides, new sections were cut from the paraffin-embedded tissue for immunohistochemistry using an avidin-biotin peroxidase method with a combination of three EHV-1/-4 crossreactive monoclonal antibodies (van Maanen et al., 2000). A minimum of four spinal cord and four brain sections from each horse were evaluated. Liver from a documented EHV-1-aborted foetus served as a positive control for the immunohistochemistry.

\section{Sample analysis}

Viral isolation and titration of nasal swabs were performed at the Virology Unit RIVM in Bilthoven, the Netherlands. All qPCR analyses and the infectious centre assays (ICA) of PBMC were conducted at the Virology Division, Department of Infectious Diseases and Immunology, Faculty of Veterinary Medicine, Utrecht University, the Netherlands. SN titres and serology testing using EHV-1 gG specific ELISA were done at the Department of Diagnostic Research and Epidemiology, Animal Health Service, Deventer, the Netherlands. The CF assay was performed at the Virology Unit, The Irish Equine Centre, Johnstown, Ireland, and the albumin measurements in CSF and serum were done at the Department of Clinical Chemistry, University Medical Centre, Utrecht, The Netherlands.

Nasal swabs were thawed, placed in $3 \mathrm{~mL}$ DMEM and vortexed. One millilitre of the suspension was applied onto rabbit kidney (RK) 13 cells for virus isolation, to be followed by virus titration as needed. Plates were inspected daily for cytopathic effects. PBMC in methylcellulose, $2.5 \times 10^{6}$ cells per assay, were seeded in duplicate onto RK13 cells in an ICA (Dutta and Myrup, 1983). The plates were inspected daily for cytopathic effects for 5 days. Serial nasal swab extract and serial PBMC from all four horses were submitted for qPCR analysis. Serial plasma samples from horses $C$ and D; all lumbo-sacral CSF samples from horses A, B, and C; and post mortem CSF samples from all horses were also submitted for qPCR analysis. The DNA of a sample was extracted with a QIAmp DNA Mini Kit (Qiagen, Westburg BV).

The following volumes from samples were used for DNA extraction: $200 \mu \mathrm{L}$ from nasal swab extracts, CSF and blood plasma and $2.5 \times 10^{6}$ PBMC in $200 \mu \mathrm{L}$ RPMI-1640. The DNA was eluted into $200 \mu \mathrm{L}$ of buffer, with the exception of DNA from CSF samples which was eluted into $100 \mu \mathrm{L}$ of buffer. An EHV-1-specific qPCR using an ABI Prism 7000 Sequence Detection System (Applera Nederland B.V.) was used to detect a DNA sequence of the EHV-1 glycoprotein $B(\mathrm{gB})$. A serial dilution of a transfected plasmid carrying the EHV-1 gB DNA sequence at a known concentration allowed quantitative assessment, and results were reported as viral genomic copy numbers. The principle of EHV-1 qPCR has been described by Hussey et al. (2006); however, the assay used here had different primers and probe nucleotide sequences (forward primer: AAGTAGAATAGCAACTGCGTGGTGT; Tm: $55.9^{\circ} \mathrm{C}$; reverse primer: GCTCGGGTTAATCTTCACCATTT; Tm: $53.3^{\circ} \mathrm{C}$; probe: 6-FAMCAAAGAGCGGACCCTAT-TAMRA; Tm: $46.8^{\circ} \mathrm{C}$ ) with detection limits at 35 genomic copies per reaction (Kirisawa et al., 1993; Goehring, 2005).

Immediate pre- and post-infection serum samples were analysed for the presence of EHV-1-specific immunoglobulins using EHV-1 gG specific ELISA and a CF assay (Hartley et al., 2005). For AQ calculations, albumin concentrations in CSF and serum against an equine albumin standard (Equine Albumin, Sigma-Aldrich) were measured with a nephelometer (BN II, Dade Behring BV) (Goehring et al., 2006b) using the following formula: CSF albumin concentration/serum albumin concentration $\times 100$. Normal horse AQ was assumed at 0.8-2.2 (Andrews et al., 1994). A CSF cell count was done manually using a Fuchs-Rosenthal cytometer. Xanthochromia was assessed subjectively by placing the sample in front of a white background, and comparing it to water. Xanthochromia was graded 0 , no difference from control; 1 , mild yellow discoloration; or 2, distinct yellow discoloration.
All experiments on living horses were performed according to, following guidelines of and with permission (DEC0204.0605) from the Animal Welfare Committee of Utrecht University, The Netherlands.

\section{Results}

\section{Clinical disease, viral nasal shedding and viraemia post-infection}

All four test horses showed clinical signs of primary EHV-1 infection. These included fever (Fig. 1), inappetence, serous nasal discharge and mild mandibular lymphadenopathy. All four horses urinated and defecated normally. While horses A and B maintained a fair tail tone, horses $C$ and $D$ had a weak tail tone during the primary fever or immediate post-infection period.

Neither of the reserve/control horses responded with a fever or clinical abnormalities to the nebulised virus culture medium suspension, and both remained clinically normal until the end of the study.

On D8, horse C developed a cell-associated viraemia and was found with a fever on D9. While the fever persisted until the end of the study, viraemia was only detectable until D12 (Figs. 1 and 2 ). Despite shedding large amounts of virus from the nasal passages, none of the other horses developed a secondary fever or measurable cell-associated viraemia.

Mild gait deficits in horse C began between D2 and D3, and were initially attributed to fever and general malaise in the immediate post-infection period. However, marked and easily detectable ataxia and weakness in the fore- and hind-limbs developed by D13. The onset of the neurological deficits coincided with cessation of measurable viraemia and worsened significantly over the next 24 h (Fig. 2).

Horses A and D had extremely mild and transient clinical signs of neurological disease, having only inconsistent weakness and ataxia (Fig. 2). Horse B showed only mild weakness on D8 and was not considered abnormal. The clinical signs in horse A started on D7 when it needed assistance to rise and lasted 4 days. Horse D began to show neurological symptoms on D3 which lasted throughout the study. Horse D had intermittent muscle fasciculations of the neck and rump, and mild dysmetria with intermittent sweating. These signs worsened with stress, excitement and when it heard the horse in the other unit. These signs were considered atypical for EHM.

Post-infection changes in the CSF occurred in horses B and C, but EHM-typical changes only occurred in horse C. Unfortunately an ascending bacterial infection of the sub-arachnoidal catheter in horse B led to its early removal on D9 and CSF could no longer be collected.

The CSF of horse C became mildly xanthochromic between D3 and D5 and had the colour of dilute urine. Between D6 and D11 it became markedly xanthochromic; however, all CSF samples remained acellular (Fig. 3). After D11 the sub-arachnoidal catheter was removed from this horse because of a suspected catheter infection and no further CSF could be collected until after euthanasia. The CSF albumin concentrations in horse $C$ remained low throughout the study, with $A Q$ within the reference range (Fig. 3). In the post mortem CSF sample, marked xanthochromia without pleocytosis was still noted.

Histopathology of the spinal cord showed approximately 1-2 distinct lesions in each cut-section. The lesions were considered typical for EHM in both the white and grey matter of the spinal cord. Immunohistochemical staining of the samples was negative for EHV-1 antigen. Using qPCR, virus was not detected in the CSF or CNS tissue of horse $C$ except for in the rete mirabilis.

None of the CSF samples collected from horse A or B indicated that there had been EHM (Fig. 3). The results of the analysis of CSF taken from horse B on D4 showed a neutrophilic pleocytosis, 

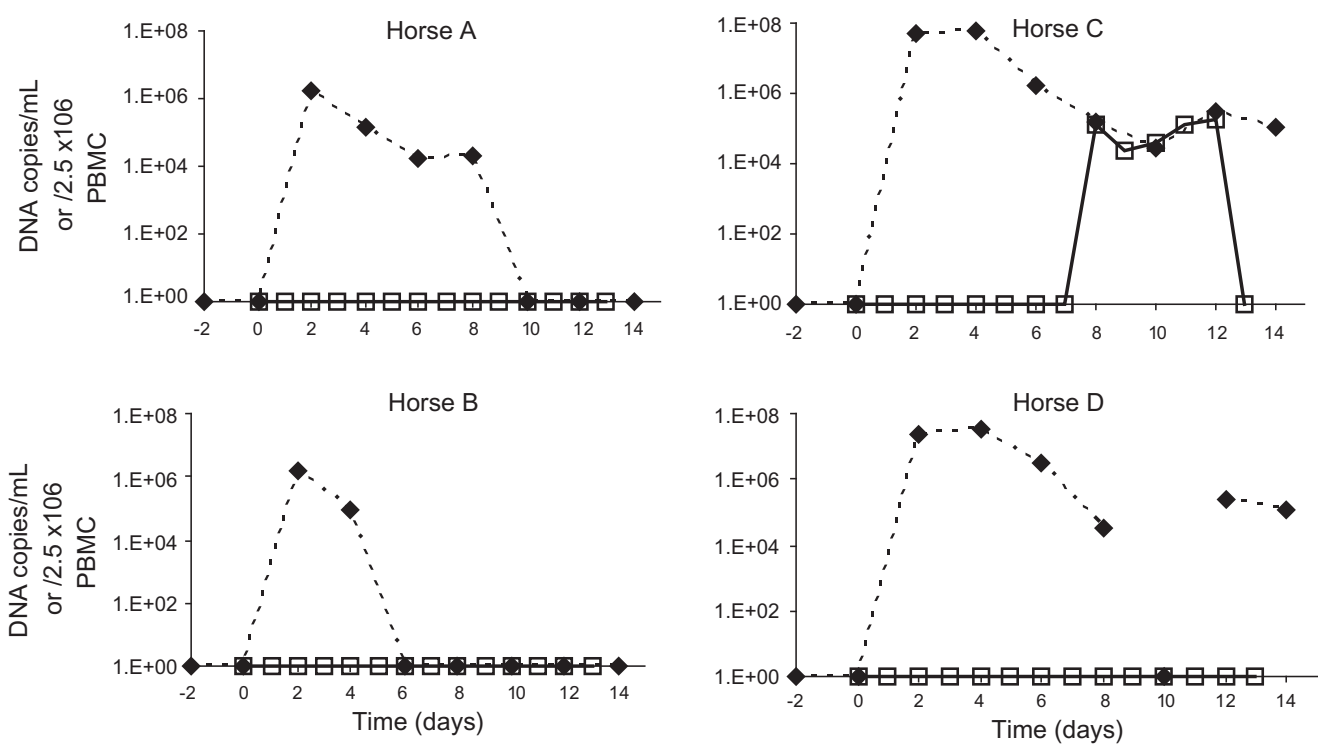

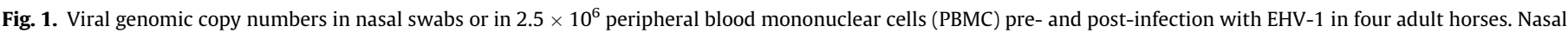

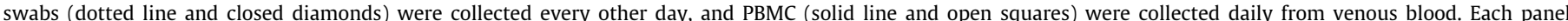

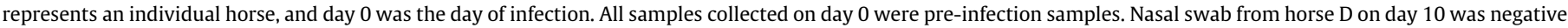
upon analysis.
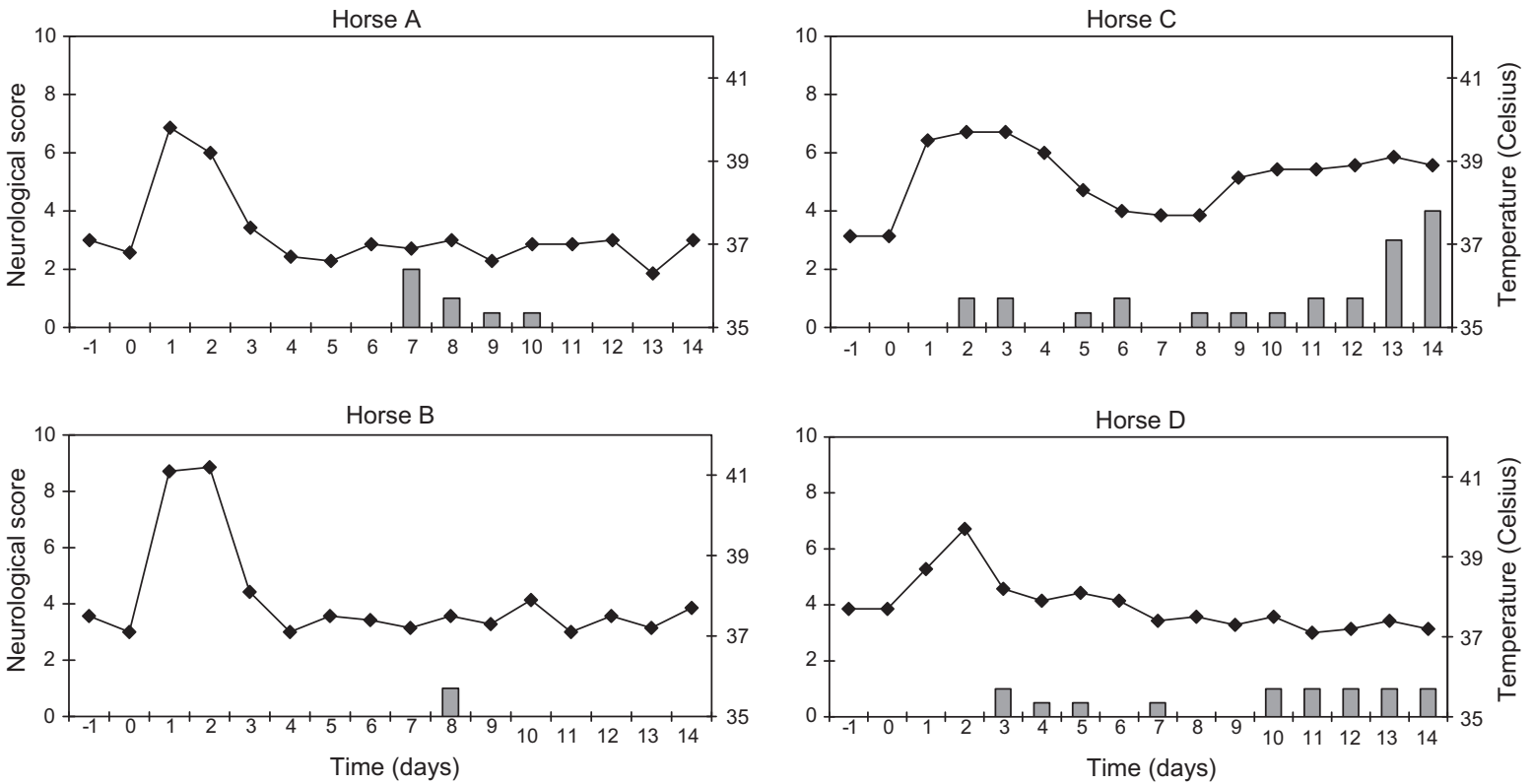

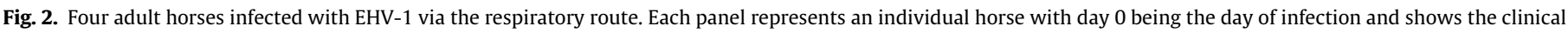

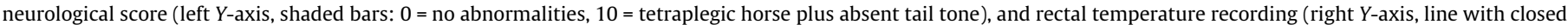
diamonds: in degrees celsius, normal: $\left\langle 38.5^{\circ} \mathrm{C}\right.$ ). Morning findings are presented.

slight turbidity and mild xanthochromia that were most consistent with bacterial meningitis. This was considered to be due to the ascending infection along the sub-arachnoidal catheter; however, because the leucocyte count in CSF had initially normalised after some adjustments of the catheter itself, the catheter was left in place. By D9 the pleocytosis reoccurred and the catheter was immediately removed. Horse B was then started on trimethoprim-sulfamethoxazole treatments. Post mortem analyses of CSF and spinal cord histopathology from horses A, B and D were all normal.

\section{Serology results, $q P C R$ and virus culture results}

Successful infection with EHV-1 was shown by specific antibody titre increases in CF assay and EHV-1 gG specific ELISA in serum samples from all four horses collected by D14. Complement fixating antibodies showed a faster and more pronounced increase compared to EHV-1 gG specific antibodies (Fig. 4). The final serum sample (D14) collected from horse C was just barely considered positive according to manufacturer's instructions for the EHV-1 gG specific ELISA. The qPCR results indicated that all infected 

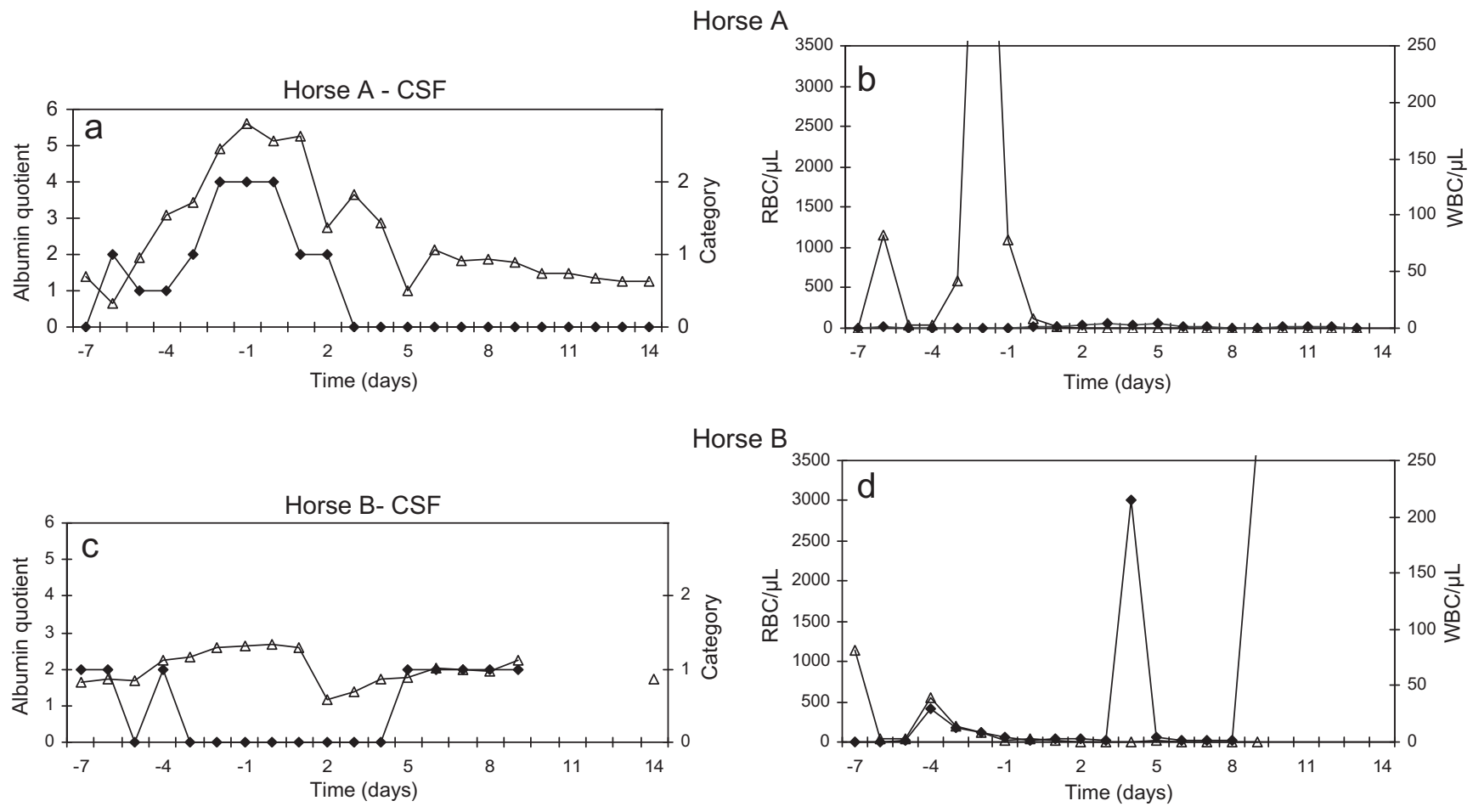

Horse C
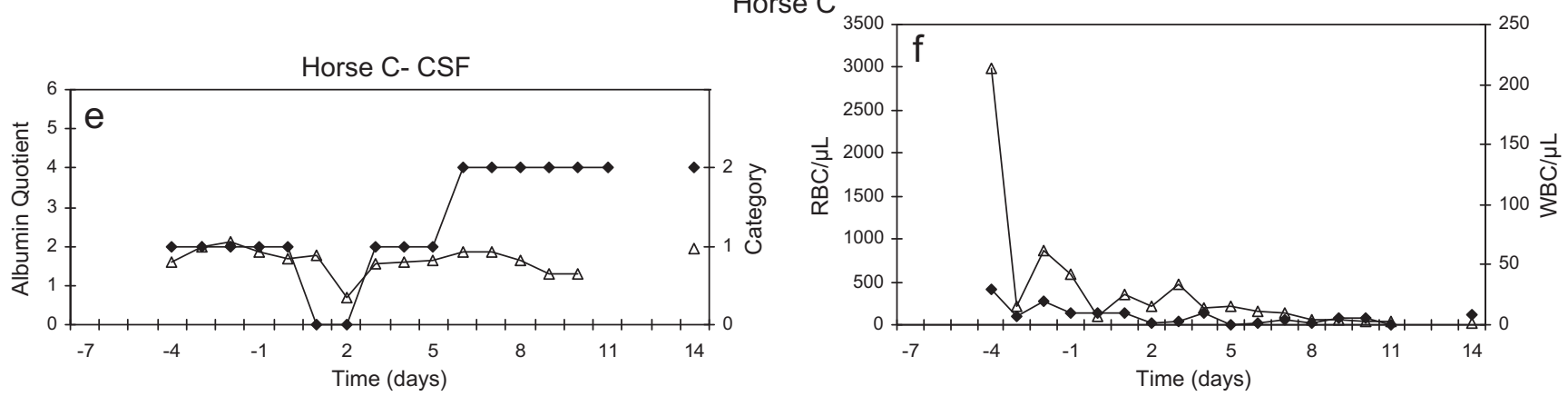

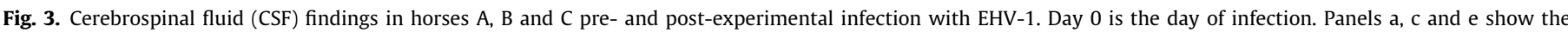

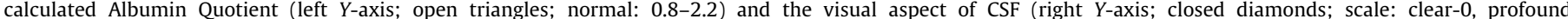

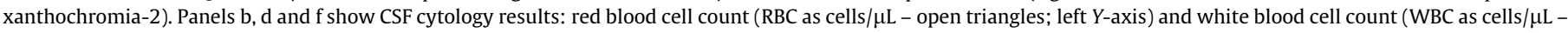
closed diamonds; right $Y$-axis).

horses' nasal passages contained large numbers of EHV-1 gB copies. This presence was of shorter duration in horses A and B ( 6 and 10 days, respectively), than in horses $C$ and $D$ in which viral (gB) genomic copies were detectable throughout the study (Fig. 1).

Unexpectedly, virus isolation from nasal swabs was generally unsuccessful and a virus titration could not be done. Only one PBMC sample collected from horse C on D9 was positive for EHV-1 in an ICA. The positive ICA result corresponded with positive qPCR results for EHV-1 gB copies (Fig. 1). Plasma fractions from horses $C$ and D did not reveal viral DNA, and neither did one of the collected CSF samples. From all the evaluated tissue sections genomic EHV-1 DNA could only be detected in the rete mirabilis in horse $\mathrm{C}$.

\section{Discussion}

A conclusive case of EHM developed only in the horse that demonstrated cell-associated viraemia, with the clinical-neurological findings supported by characteristic CSF changes and histopathological findings on examination of the horse's spinal cord. Positive immunohistochemistry of the lesions for EHV-1 antigen would have been ideal, but this required the expression of viral proteins by the infected cell, which does not always occur (Slater, 2008).

The results of this study provide further support that the EHV-1 cell-associated viraemia is a necessary pre-requisite for EHM, although no inferences could be made about the relationship between the severity of clinical-neurological signs and the quantity and duration of viraemia. Non-cell-associated or 'free' EHV-1 viraemia was also tested for but only in post-EHV-1 infection plasma samples from two horses with the prolonged viral nasal shedding ( $C$ and $D$ ) and all samples were negative. However, this may be due to the small sample size $(200 \mu \mathrm{L})$ and further studies collecting larger volumes of plasma each day are necessary to determine if 'free viraemia' occurs.

Surprisingly, 3/4 EHV-1 seronegative, age-, breed- and sexmatched horses that underwent an identical inoculation with EHV-1 did not develop a qPCR-detectable cell-associated viraemia. We assumed that a strong, protective antigen-specific immunological threshold must have existed at the moment of infection, which suppressed cell-associated viremia beyond levels of detection. All EHV-1 infection studies prior to 2006 that used ponies or horses, 

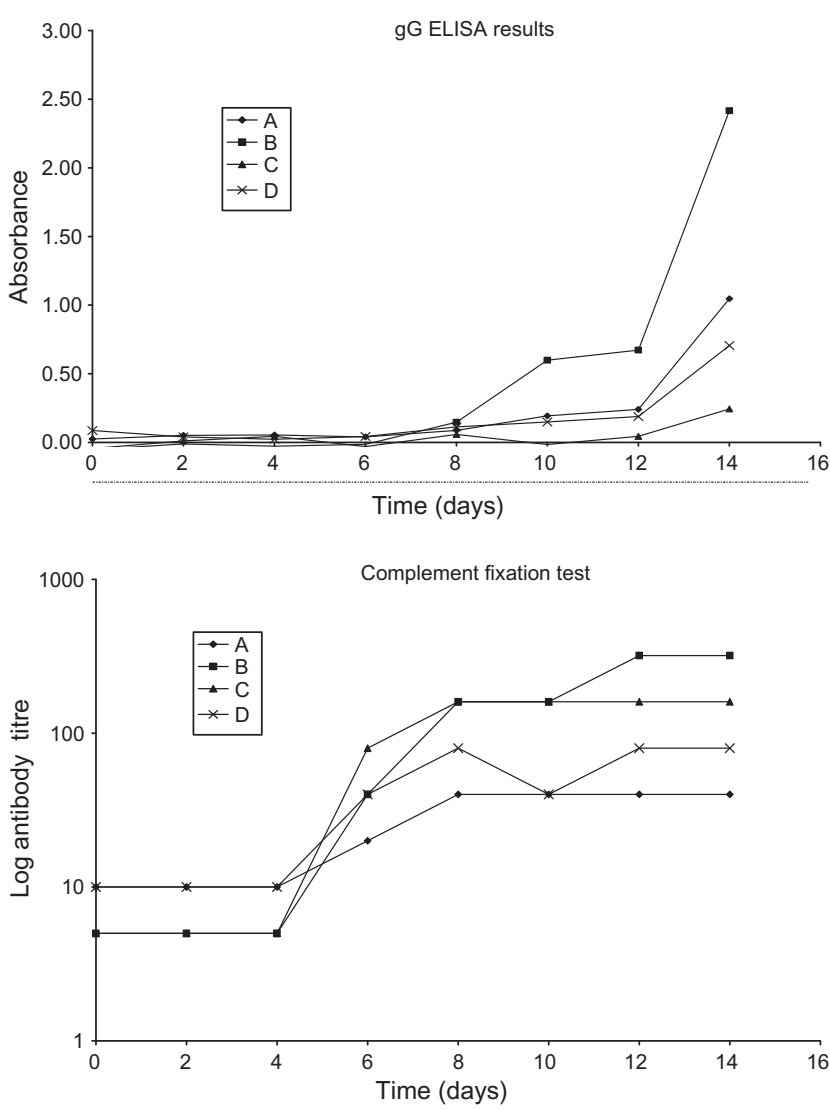

Fig. 4. Serology results of an EHV-1 infection experiment in four horses. Day 0 is the day of infection. Upper panel: antibody concentration against EHV-1 gG, expressed as absorbance (A415 nm) in serum samples pre- and post-infection. An absorbance of $<0.2$ is a negative sample, and this cut-off is represented by a dotted line. Lower panel: complement fixing antibody titer against EHV-1 in serum samples pre and post-infection. A titre $<1: 10$ is considered 'negative'.

yearlings or middle-aged animals and different EHV-1 strains for infection selected their experimental animals based upon absent evidence of a recent EHV-1 exposure. Typically, the selection was based on serological testing using an EHV-1 SN test or a CF assay (Mumford et al., 1994; Goodman et al., 2006).

Selecting horses for our infection study used inclusion criteria with regard to antibody testing, in which we used three different tests for EHV-1 antibody detection, an SN test, a CF test and the EHV-1 gG specific ELISA (Hartley et al., 2005). The SN test was used for screening purposes, but only identified six horses with the lowest EHV-1 SN titre possible. The problems with this test include cross-reactivity to the more common EHV-4 strain and SN titre results may vary between laboratories due to protocol differences. High EHV-1 SN titres can be found for months following infection or vaccination (Mumford et al., 2003). The same is true for an antibody titre measured with the EHV-1 gG specific ELISA; however, the antibodies measured with this test are EHV- 1 specific. A titre in the CF assay indicates recent infection. However, similar to the EHV-1 SN test, a CF assay will also measure complement-binding, cross-reactive antibodies produced against EHV-4. It is therefore possible that, despite our efforts to rule out EHV-1 and EHV-4 infection prior to and during our study, a preventative immunemechanism may have been present in the three apparently nonviraemic horses.

Our results suggested that there was a high frequency of precursor antigen-specific cytotoxic T lymphocytes (CTL) present in the non-viraemic horses, which is an immune-mechanism considered among the most powerful tools for minimising cell-associated
EHV-1-viraemia. CTL presence is thought to last longer than the half-life of antibodies (Kydd et al., 2003, 2006). Unfortunately, the test used to measure specific CTL frequencies during this study, a chromium release assay, was only available in few highly specialised research laboratories. In addition, the assay requires a large number of PBMC, which were not available from our study. However, results of the antigen-specific CTL frequency in the three non-viraemic horses would have been of great value to provide a most likely argument for the absence of viraemia.

Recently, Allen (2009) provided convincing evidence of an inverse correlation between the magnitude of EHV-1 viraemia and the frequency of EHV-1 antigen-specific precursor CTL. Future infection studies that focus on the infection of middle-aged to aged horses, which are likely to have been previously exposed to EHV-1, will need to determine the EHV-1-specific precursor CTL frequencies as a part of the horse selection. A high CTL frequency may prevent a cell-associated viraemia and, due to a reduced or even complete lack of CNS endothelial cell infection, it may prevent the induction of neurological disease (Allen, 2009).

An alternative explanation for the absence of measurable cellassociated viraemia in horses A, B, and D could be due to variations in the individual infectious doses applied to each horse by closed inhalation. This could be reflected in the differences in duration of viral nasal shedding post-infection. However, the viral inoculum used was harvested from cell culture flasks, split into four equal fractions, frozen, and then thawed prior to infection, and no procedural differences in infection by nebuliser and mask were identified. Also the primary clinical signs of a horse to infection were similar in all horses.

Viral isolation from nasal swabs was unsuccessful in this study. The discrepancy between high viral genomic copy numbers in nasal swabs, as measured by qPCR, and unsuccessful virus isolation may suggest that severely damaged virus was used as inoculum, which allowed the detection of DNA, but not virus isolation. However, two arguments disqualify these assumptions. First, in retrospect, it was found that due to miscommunication, significant mistakes were made by freezing nasal swabs in empty containers instead of immersing the swabs in appropriate virus transportation medium prior to freezing. Secondly, after a period of proper storage temperature at $-80^{\circ} \mathrm{C}$, the nasal swabs were stored at $-20^{\circ} \mathrm{C}$ for more than 6 weeks. Because both procedural mistakes would have interfered with viral envelope integrity, a crucial instrument for host cell infection, it is the most likely explanation for negative virus isolation results. In addition, the titre of the inoculum that had been given to each horse $\left(10^{7.6} \mathrm{TCID}_{50} / \mathrm{mL}\right)$ was determined from the fluid remaining in the nebulisation chamber after administration, and the genomic copy numbers we found in the postinfection period in the horses in this study were very similar to those measured by Perkins et al. (2008) in horses experimentally infected with EHV-1. Perkins et al. (2008) showed that there was a positive relationship between genomic copy numbers and virus isolation results on nasal swabs.

Horse $\mathrm{C}$ had a secondary fever (a finding often associated with viraemia; Goehring et al., 2008), clear signs of neurological disease, and CSF and histopathological indicators of EHM. However, horses A and D also showed mild, transient or intermittent signs of neurological disease without measurable cell-associated viraemia or secondary fever. The neurological deficits in these two horses were graded as low as 0.5 or 1 out of a possible 10 , which are extremely mild abnormalities. Horse A was scored once with $2 / 10$ on D7, because it needed assistance to stand up once, and it showed mild ataxia/weakness afterwards (Fig. 2).

Clinical-neurological examinations are a subjective assessment of nervous system function. Although the morning and evening neurological evaluations were conducted by two different veterinarians, one of them being very familiar with the neurological 
examination of horses, the level of agreement on abnormal findings was $>90 \%$. However, assessment in the post-infection period was slightly hampered by lack of space in which to perform a thorough neurological examination; in particular, the inability to walk a horse on a straight line over a certain distance. Despite the examination limitations, the investigators felt that they achieved a high level of sensitivity for detecting abnormalities. However, determination that these clinical findings were truly the result of neurological disease was probably less accurate. Findings such as 'weakness', 'decreased tail tone', and 'mild ataxia' may have been the result of a fever or general malaise after infection and these findings may have been over-interpreted.

CSF evaluation was used in horse A and histopathological evaluation of nervous tissue was used in horses $A$ and $D$ to distinguish between structural nervous system disease and potentially falsepositive clinical exam results. Neither of these animals showed histopathological abnormalities. In fact, acute or recent damage to the spinal cord was only seen in horse $C$, although immunohistochemical staining for EHV-1 (and -4) antigen of the tissues were negative.

The interpretation of the mild clinical-neurological abnormalities in horses A and D with undetectable viraemia and normal histopathology and normal CSF analyses in horse A remains unclear. These findings may have been false-positive findings. Another explanation potentially is that the amount of virus in PBMC may have been below the PCR detection limits and/or that affected sites in the spinal cord may have been missed on sectioning since they were assumed fewer and less severe based on the mild clinical signs that were noted. For future experiments it is strongly recommend to sample spinal cord cross-sections more frequently than one section every $10 \mathrm{~cm}$. Small lesions can be focal and only visible microscopically. Alternatively, it is possible that the time between cessation of clinical-neurological signs and pathological examination may have been sufficient for tissue restoration. This implies the need for early, readily available and objective indicators of nervous system disease.

Although maintenance of the sub-arachnoidal catheters in the horses was challenging, we found them to be extremely valuable. Twice daily CSF analyses served as an ongoing assessment of spinal cord integrity, and the CSF analysis results were very helpful in interpreting clinical findings. CSF cell counts and the macroscopic aspect of the fluid were the easiest to perform and also the most informative parameters tested. The presence of xanthochromia in horse C was an early change in the CSF appearance that, in retrospect, should have been interpreted as an indicator for the start of progressive myelopathy. The AQ was a poor predictor of onset and progression of EHM, but it may be of greater value if more pathology develops in an individual horse. Other factors, such as interleukins, cytokines or proteins suggestive of vasculitis, ischaemia or neuronal degeneration, may also be key markers in EHM and would be important for assessing the correlation between the quantitative aspects of viraemia and the degree of (sub)clinical EHM. Future studies that focus on early CNS pathological changes could benefit from the here described CSF collection as a sudden indicator of disease. A secondary fever and viraemia are further timely indicators for the potential occurrence of EHM.

\section{Conclusions}

This experimental model for reproducing EHM in horses with a presumed increased risk of developing neurological disease resulted in only moderate disease in $1 / 4$ infected horses. We were unable to reproduce the high percentage and the case severity of EHM-affected horses reported in a series of infection experiments conducted by Mumford et al. (1994). Since a viraemia is essential to the development of EHM, a future requirement for experimental EHM studies will be to select horses with a low frequency of precursor antigen-specific CTL, as their number is inversely related to duration and magnitude of EHV-1 viraemia (Allen, 2009).

Repetitive CSF sampling provided valuable information during the prodromal stages of EHM and for detecting moderate EHM. EHM developed in association with and shortly after cessation of cell-associated viraemia, and viraemia was manifested by secondary fever following the initial post-infection fever spike. We believe that the findings of this study add to the knowledge of the pathogenesis of EHM, many aspects of which remain unclear. Although this study demonstrated the usefulness of repetitive CSF sampling, the analysis of the collected sample may require more sensitive assays to detect early or mild neuropathology. Additional testing such as a CTL frequency measurement during selection of horses for an infection study and the more sensitive monitoring using repetitive CSF sampling during an infection study will lead to the refinement of experimental EHV-1 infection in horses, while a histopathological examination needs to be the base of an infection model.

\section{Conflict of interest statement}

None of the authors of this paper has a financial or personal relationship with other people or organisations that could inappropriately influence or bias the content of the paper.

\section{Acknowledgements}

The authors wish to thank Dr. Janneke Sterk, Martin van Leeuwen, Jan Stolwijk and Marjory Pollak for excellent technical assistance; Dr. Ted van den Ingh for providing histopathology and immunohistochemistry results; Dr. Nick Davis-Poynter for characterising the EHV-1 strain; and Daniel Salamon and Dr. Mary E. Carlson for critically editing this script.

\section{References}

Akens, M.K., Holznagel, E., Franchini, M., Bracher, V., 1997. Comparative analysis of equine lymphocyte subsets in whole blood and gradient-purified samples. Veterinary Immunology and Immunopathology 58, 231-237.

Allen, G.P., 2009. Risk factors for the development of neurologic disease after experimental exposure to equine herpesvirus-1 in horses. American Journal of Veterinary Research 69, 1596-1600.

Allen, G.P., Breathnach, C.C., 2006. Quantification by real-time PCR of the magnitude and duration of leucocyte-associated viraemia in horses infected with neuropathogenic vs. non-neuropathogenic strains of EHV-1. Equine Veterinary Journal 38, 252-257.

Andrews, F.M., Geiser, D.R., Sommardahl, C.S., Green, E.M., Provenza, M., 1994 Albumin quotient, IgG concentration, and IgG index determinations in cerebrospinal fluid of neonatal foals. American Journal of Veterinary Research $55,741-745$.

Donaldson, M.T., Sweeney, C.R., 1998. Herpesvirus myeloencephalopathy in horses: 11 cases (1982-1996). Journal of the American Veterinary Association 213 671-675

Dutta, S.K., Myrup, A.C., 1983. Infectious center assay of intracellular virus and infective virus titer for equine mononuclear cells infected in vivo and in vitro with equine herpesviruses. Canadian Journal of Comparative Medicine 47, 6469.

Edington, N., Bridges, C.G., Patel, J.R., 1986. Endothelial cell infection and thrombosis in paralysis caused by equid herpesvirus-1: equine stroke. Archives of Virology 90, 111-124.

Goehring, L.S., 2005. Equine herpesvirus-associated myeloencephalopathy: aspects of epidemiology and pathogenesis. Thesis. Department of Equine Sciences and Department of Immunology and Infectious Diseases, Utrecht University, Utrecht, The Netherlands, $165 \mathrm{pp}$.

Goehring, L.S., van Winden, S.C., van Maanen, C., Sloet van OldruitenborghOosterbaan, M.M., 2006a. Equine herpesvirus type 1-associated myeloencephalopathy in The Netherlands: a four-year retrospective study (1999-2003). Journal of Veterinary Internal Medicine 20, 601-607.

Goehring, L.S., Kessels, B.G., van Maanen, C., Voorbij, H.A.M., Sloet van Oldruitenborgh-Oosterbaan, M.M., 2006b. Evaluation of nephelometry for albumin measurement in serum and cerebrospinal fluid: experiences with an 
indwelling subarachnoidal catheter system for repetitive cerebrospinal fluid collection in horses. Journal of Veterinary Diagnostic Investigation 18, 251-256.

Goehring, L.S., Hussey, S.B., Rao, S., Bigbie, R., Lunn, D.P., 2008. Control of EHV-1 viremia and nasal shedding by current commercial vaccines. Abstract \#124 26th ACVIM Forum, San Antonio, Texas. Journal of Veterinary Internal Medicine $22,737-738$.

Goodman, L.B., Wagner, B., Flaminio, M.J.B.F., Sussman, K.H., Metzger, S.M., Holland R., Osterrieder, N., 2006. Comparison of the efficacy of inactivated combination or modified-live virus vaccines against challenge infection with neuropathogenic equine herpesvirus type 1 (EHV-1). Vaccine 24, 3636-3645.

Hartley, C.A., Wilks, C.R., Studdert, M.J., Gilkerson, J.R., 2005. Comparison of antibody detection assays for the diagnosis of equine herpesvirus 1 and 4 infections in horses. American Journal of Veterinary Research 66, 921-928.

Henninger, R.W., Reed, S.M., Saville, W.J., Allen, G.P., Hass, G.F., Kohn, C.W., Sofaly, C. 2007. Outbreak of neurologic disease caused by equine herpesvirus-1 at university equestrian center. Journal of Veterinary Internal Medicine 21, 157165.

Hussey, S.B., Clark, R., Lunn, K.F., Breathnach, C., Whalley, J.M., Lunn, D.P., 2006 Detection and quantification of equine herpesvirus-1 viremia and nasa shedding by real-time polymerase chain reaction. Journal of Veterinary Diagnostic Investigation 18, 335-342.

Jackson, T.A., Osburn, B.I., Cordy, D.R., Kendrick, J.W., 1977. Equine herpesvirus 1 infection of horses: studies on the experimentally induced neurologic disease. American Journal of Veterinary Research 38, 709-719.

Kirisawa, R., Endo, A., Iwai, H., Kawakami, Y., 1993. Detection and identification of equine herpesvirus- 1 and -4 by polymerase chain reaction. Veterinary Microbiology 36, 57-67.

Kydd, J.H., Wattrang, E., Hannant, D., 2003. Pre-infection frequencies of equine herpesvirus-1 specific, cytotoxic $\mathrm{T}$ lymphocytes correlate with protection against abortion following experimental infection of pregnant mares. Veterinary Immunology and Immunopathology 96, 207-217.

Kydd, J.H., Townsend, H.G., Hannant, D., 2006. The equine immune response to equine herpesvirus-1: the virus and its vaccines. Veterinary Immunology and Immunopathology 111, 15-30.
Mayhew, I.G., 2008. Neurologic evaluation. In: Large Animal Neurology, second ed. Wiley-Blackwell, Ames, Iowa, pp. 11-46.

Mumford, J.A., Hannant, D., O’Neill, T., Smith, K.C., Ostlund, E.N., 1994. Abortigenic and neurological disease caused by experimental infection with equid herpesvirus-1. In: Proceedings of the 7th International Conference on Equine Infectious Diseases, Tokyo, Japan, pp. 261-275.

Mumford, E.L., Traub-Dargatz, J.L., Carman, J., Callan, R.J., Collins, J.K., Goltz, K.L., Romm, S.R., Tarr, S.F. Salman, M.D., 2003. Occurrence of infectious upper respiratory tract disease and response to vaccination in horses on six sentinel premises in northern Colorado. Equine Veterinary Journal 35, 72-77.

Nugent, J., Birch-Machin, I., Smith, K.C., Mumford, J.A., Swann, Z., Newton, J.R., Bowden, R.J., Allen, G.P., Davis-Poynter, N., 2006. Analysis of equid herpesvirus 1 strain variation reveals a point mutation of the DNA polymerase strongly associated with neuropathogenic versus nonneuropathogenic disease outbreaks. Journal of Virology 80, 4047-4060.

Perkins, G.A., Goodman, L.B., Dubovi, E.J., Kim, S.G., Osterrieder, N., 2008. Detection of equine herpesvirus-1 in nasal swabs of horses by quantitative real-time PCR. Journal of Veterinary Internal Medicine 22, 1234-1238.

Slater, J.D., 2008. Equine herpesviruses. In: Sellon, D.C., Long, M.T. (Eds.), Equine Infectious Diseases. Saunders Elsevier, St. Louis MO, USA, pp. 134-153.

Smith, D.J., Hamblin, A.S., Edington, N., 2001. Infection of endothelial cells with equine herpesvirus-1 (EHV-1) occurs where there is activation of putative adhesion molecules: a mechanism for transfer of virus. Equine Veterinary Journal 33, 138-142.

van Maanen, C., 2002. Equine herpesvirus 1 and 4 infections: an update. Veterinary Quarterly 24, 58-78.

van Maanen, C., de Boer-Luijtze, E., Terpstra, C., 2000. Development and validation of a monoclonal antibody blocking ELISA for the detection of antibodies against both equine herpesvirus type 1 (EHV1) and equine herpesvirus type 4 (EHV4). Veterinary Microbiology 71, 37-51.

van Maanen, C., Sloet van Oldruitenborgh-Oosterbaan, M.M., Damen, E.A., Derksen, A.G.P., 2001. Neurological disease associated with EHV-1-infection in a riding school: clinical and virological characteristics. Equine Veterinary Journal 33, 191-196. 